\title{
Partial Discharge Patterns in High Voltage Insulation
}

\author{
Hazlee Illias, Teo Soon Yuan, Ab Halim Abu Bakar, \\ Hazlie Mokhlis \\ Electrical Engineering Department, UMPEDAC, \\ Faculty of Engineering, University of Malaya \\ Kuala Lumpur, Malaysia \\ h.illias@um.edu.my
}

\author{
George Chen, Paul L. Lewin \\ The Tony Davies High Voltage Laboratory \\ School of electronics and Computer Science \\ University of Southampton \\ Southampton, United Kingdom
}

\begin{abstract}
Partial discharge (PD) is an electrical discharge, which does not bridge the electrodes between an insulation system completely under high electric field stress. PD events may affect the performance of insulation system because in long term, it may cause the breakdown of insulation system and failure of high voltage component due to repetition of PD events. In this work, two techniques of analysing PD data have been studied; the phase resolved partial discharge (PRPD) pattern and pulse sequential analysis (PSA) techniques. These two techniques were used to distinguish three main types of PD; they are corona, void and surface discharges. PD data were obtained from the measurement in high voltage laboratory in the University of Southampton, UK. In the end of the analysis, comparison between the PRPD and PSA methods was done to evaluate the advantages and disadvantages between the two techniques.
\end{abstract}

Keywords-partial discharge; corona discharge; insulating materials, high voltage insulation

\section{INTRODUCTION}

Partial discharge (PD) is an electrical discharge event that does not bridge completely the electrodes between an insulation system under high electric field stress. Generally, PD occurs at the defect sites such as delaminations, cavities, joints or voids in insulation system of high voltage components, such as power generators, power transformers, power transmission line and power cables.

PD normally occurs at the defect sites within the insulation system because of higher breakdown strength of insulation material than the defect site [1]. Mostly, defects present in the insulation system in the form of gas-filled void during the manufacturing. However, the gas-filled void cannot be detected in the factory testing. These defects can affect the performance of the insulation system in service because the repetition of PDs causes the degradation of insulation system, which may lead to breakdown of insulation system. Consequently, failure of high voltage equipment will occur. The breakdown of insulation system requires costly and time consuming maintenance as the whole component needs to be replaced. Therefore, PD measurement and PD data analysis are essential to assess the performance and condition of insulation system in order to avoid the unwanted breakdown of insulation system. This may help in reducing the cost and time of maintenance.

There are two techniques of measuring and analysing PD activities which are widely used; they are phase resolved partial discharge (PRPD) pattern and pulse sequential analysis (PSA) techniques. These techniques can be used to distinguish the types of PD, based on the patterns obtained. The PRPD technique makes use of the phase and charge magnitude of PD occurrences [2]. The phase axis (x-axis) consists of one complete cycle of the applied voltage while the PD charge magnitude axis (y-axis) consists of the range of magnitude detected. PD data within certain number of the applied voltage cycle is plotted on the $\mathrm{x}$-axis of one voltage cycle. Therefore, a PRPD pattern shows PD occurrences at a specific phase of the applied voltage with certain charge magnitude within certain number of the applied voltage cycles.

The pulse sequential analysis (PSA) technique makes use of the applied voltage amplitude when a PD occurs and the time of the PD occurrence [3]. It does not consider the phase and charge magnitude of the PD occurrence. In a PSA pattern, the $\mathrm{y}$-axis is the voltage difference between the next and current PD occurrences while the $x$-axis is the voltage difference between the current and previous PD occurrences. It is similar for the time difference between consecutive PDs pattern. In general, PSA patterns make use of the sequence of PD occurrences.

In this work, PRPD and PSA techniques have been used to differentiate between different types of PD from the measurement that have been performed in the high voltage laboratory in the University of Southampton, UK. The experiment setup was for void, surface and corona discharges measurement. Sets of PD signals from the measurement were captured using a digital signal oscilloscope (DSO) and PRPD and PSA patterns were obtained using a MATLAB programming code.

\section{MEASUREMENT SetuP}

\section{A. Void discharge}

Figure 1 shows the test object that has been used in the measurement of void discharges. The test sample consists of a cylindrical epoxy resin of $3.5 \mathrm{~mm}$ thickness and $38 \mathrm{~mm}$ radius and a spherical void. Initially, a spherical void of $2 \mathrm{~mm}$ diameter was placed in a smaller epoxy resin block of $2.5 \mathrm{~mm}$ thickness. The spherical void was prepared by injecting a bubble in the resin. Then, the smaller block of epoxy containing the void was placed in the middle of the larger epoxy resin block. They were left to cure for 24 hours at room 
temperature, post cure for 4 hours at $90^{\circ} \mathrm{C}$ and finally cooling at room temperature. A stainless steel cylindrical electrode was placed on each of the upper and bottom surfaces of the test sample. The upper electrode was connected to the $50 \mathrm{~Hz}$ sinusoidal voltage while the lower electrode was grounded. The whole test object was immersed in mineral oil to prevent surface discharges around the edge of the electrode $[4,5]$.

\section{B. Surface discharge}

The test object that has been in the measurement of surface discharges is shown in Figure 2. A sharp pin electrode was placed on the surface of XLPE material and was applied with a $50 \mathrm{~Hz}$ sinusoidal applied voltage. The bottom surface of the material was always grounded.

\section{Corona discharge}

Figure 3 shows the test object that has been used in the measurement of corona discharges. The setup consists of a sharp end electrode, which was applied with a $50 \mathrm{~Hz}$ sinusoidal voltage and a grounded plane.

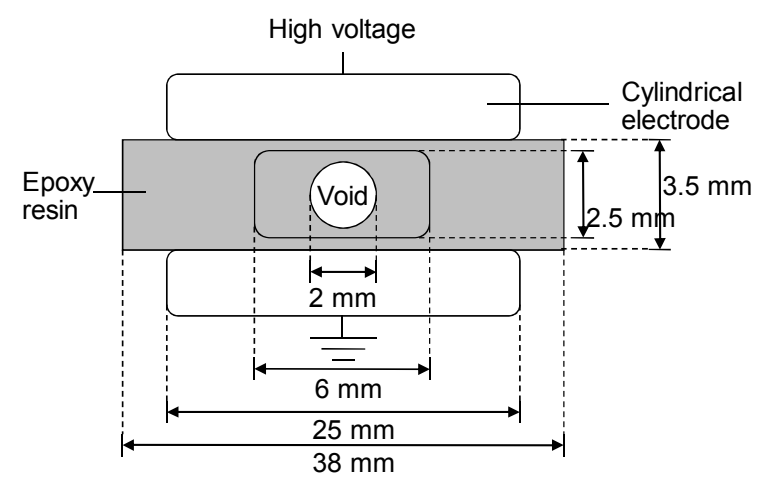

Figure 1. Test object for void discharge measurement

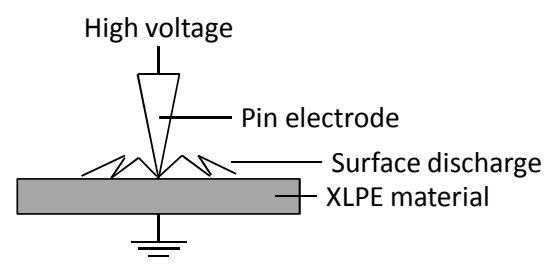

Figure 2. Test object for surface discharge experiment

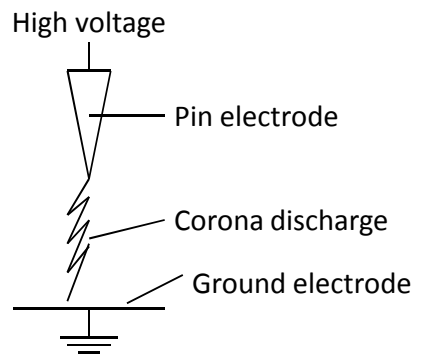

Figure 3. Test object for corona discharge experiment

\section{PD measurement setup}

The measurement setup of PD activity is shown in Figure 4 [6]. It consists of a high voltage supply, $\mathrm{V}$, a coupling capacitor, $\mathrm{C}_{\mathrm{k}}$, a test object, a coupling device, a PD detector and a USB controller, which is connected to a personal computer (PC) for data analysis. The coupling device and the PD detector are used to detect PD signals from the test object.

\section{MEASUREMENT RESUlts OF PRPD PATTERNS}

\section{A. Void discharge}

Figure 5 shows PRPD patterns of void discharge at 18 and $24 \mathrm{kV}$ sinusoidal applied voltages. When the applied voltage is higher, the numbers of PDs per cycle, total charge per cycle and the maximum magnitude of void discharge are higher. PD occurs in the void when the electric field in the void is higher than the inception field and there is an initial free electron to start an avalanche process. Since the process of having a free electron is random, the occurrence of a void discharge is also random. The electric field in the void follows the applied voltage waveform curve, which is sinusoidal. Thus, the PRPD patterns have a curvy shape which follows sinusoidal voltage waveform.

When the applied voltage is increased, the maximum PD charge magnitude is larger because the maximum electric field in the void is higher. At higher applied voltage, the electric field in the void increases faster towards the inception field. This results in more PDs to occur in one applied voltage cycle. Since the void is located in the middle of the material, the electric field on the surface of the void is symmetrical. Therefore, the PRPD patterns of void discharge at positive and negative cycles of the applied voltage are symmetrical.

\section{B. Surface discharge}

The PRPD patterns of surface discharge from the measurement are shown in Figure 6. When the applied voltage is increased, the numbers of PDs per cycle, total charge per cycle and the maximum charge magnitude become higher.

When the electric field at the surface of the electrode exceeds the breakdown strength of gas, ionisations of air near the surface of electrode occur. This is due to the tangential field on the insulation surface is high enough to cause PDs along the surface of the material. The surface discharges keep occurring until the transient activity stops. When the applied voltage is increased, the number of electrons ionisation increases and electron avalanche can grow longer along the material surface.

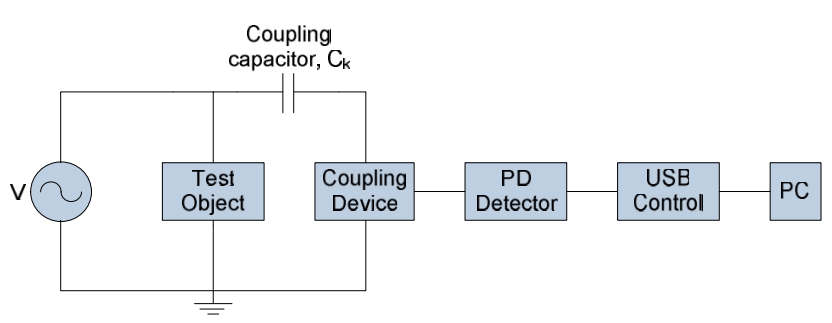

Figure 4. PD measurement setup 


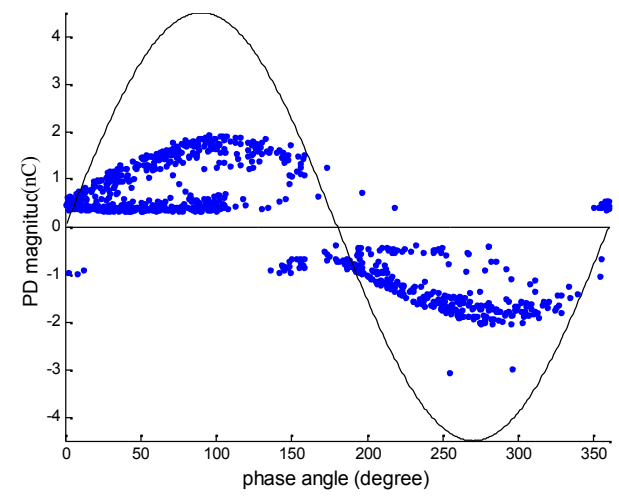

(a) $18 \mathrm{kV}$ applied voltage

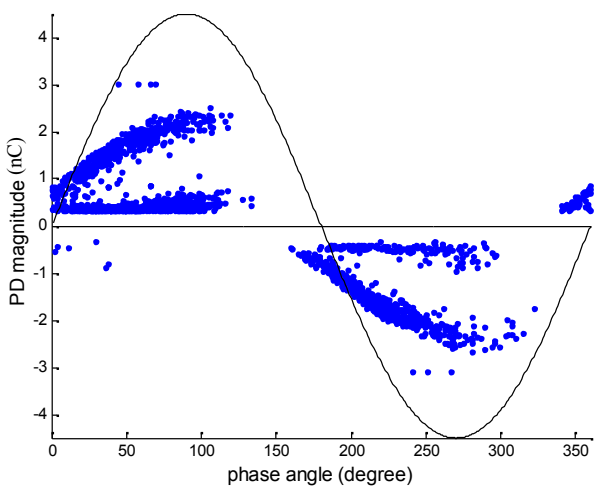

(b) $24 \mathrm{kV}$ applied voltage

Figure 5. PRPD patterns of void discharge

Referring to Figure 6, the number of PDs occurring in the positive applied voltage cycle is less than the negative applied voltage cycle. This is because more electrons are readily available from the electrode under negative applied voltage, resulting in more electron avalanches easier to be developed. The maximum PD magnitude at negative cycle is higher than at positive cycle of the applied voltage because the electric field builds up along the path of electron avalanche on the material surface. Thus, some avalanches can grow longer, yielding higher PD charge magnitude. PD stops when the electric field at the avalanche is less than the extinction field. The maximum PD charge magnitude is obtained around 270 degree phase.

From Figure 6b, PDs can be seen to occur at almost all phase of the applied voltage at negative cycle. This is due to a wide area of the material surface from the electrode for PD to occur. Thus, the probability of PD to occur is very high. Unlike void discharge PRPD patterns, the patterns of surface discharges at positive and negative applied voltage cycles are not symmetrical because the electric field at the material surface during positive and negative cycle is not symmetrical.

\section{Corona discharge}

Figure 7 shows PRPD patterns of corona discharge at 10 and $14 \mathrm{kV}$ sinusoidal applied voltages. When the applied voltage is higher, the numbers of PDs per cycle, total charge per cycle and the maximum magnitude of corona discharges are higher. This can be seen by a higher number of PDs in each applied voltage cycle at higher applied voltage.

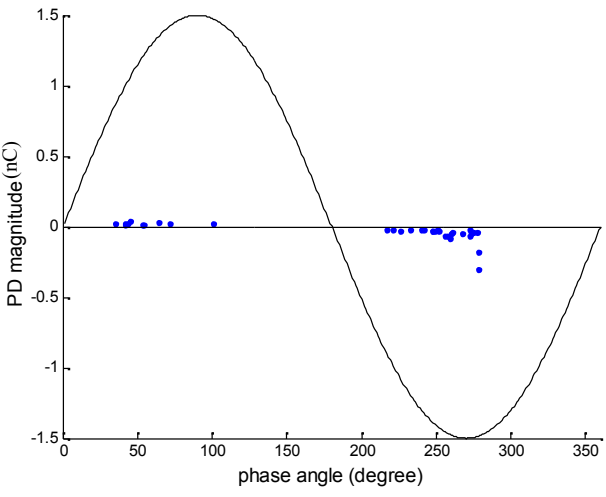

(a) $3 \mathrm{kV}$ applied voltage

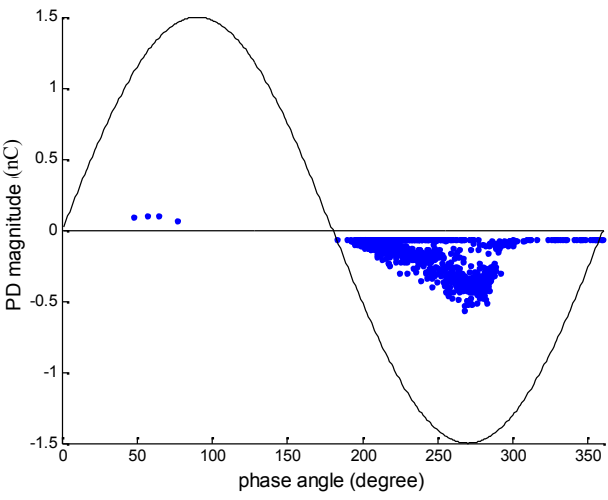

(b) $6 \mathrm{kV}$ applied voltage

Figure 6. PRPD patterns of surface discharge

When the applied voltage at the electrode (sharp point) exceeds the breakdown strength of the gas, ionisations of air near the sharp point electrode occur. Positive and negative streamers are formed depending on the applied voltage polarity. These streamers are corona discharges. Corona discharge keeps occurring until the transient activity stops, the discharge becomes self-sustained and a steady glow appears close to the anode [7]. When the applied voltage is increased, the number of streamers developed is more frequent and streamer channels can grow longer, resulting in a higher maximum PD charge magnitude. The electric field surrounding the sharp tip of the electrode increases with the applied voltage amplitude, enabling more corona PDs to occur.

From Figure 7, it can be seen that at $10 \mathrm{kV}$ applied voltage, there is no corona discharge in the positive applied voltage cycle but it appears at $14 \mathrm{kV}$. At lower applied voltage, the probability of getting an initial free electron due to the positive electrode to start an ionisation is low. Thus, no corona discharge occurs at the positive cycle of $10 \mathrm{kV}$ applied voltage. However, at higher applied voltage, the probability of an initial free electron to appear from the positive electrode is higher. Thus, positive corona discharge appears. There are many negative corona discharges occur because electrons are readily available from the negative polarity electrode to ionise the surrounding neutral gas molecules to generate avalanches. Therefore, the patterns of corona discharges at positive and negative cycles of the applied voltage are not symmetrical. 


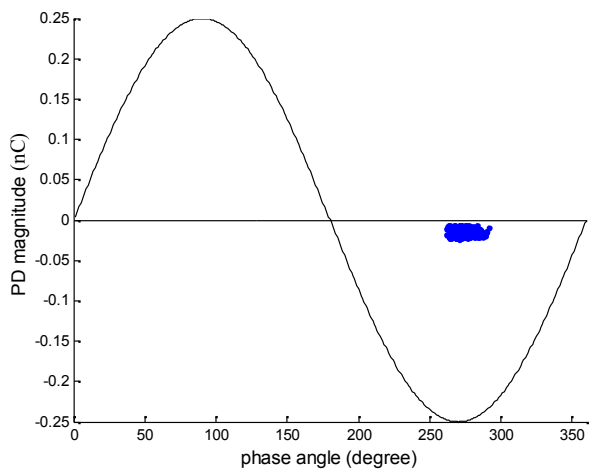

(a) $10 \mathrm{kV}$ applied voltage

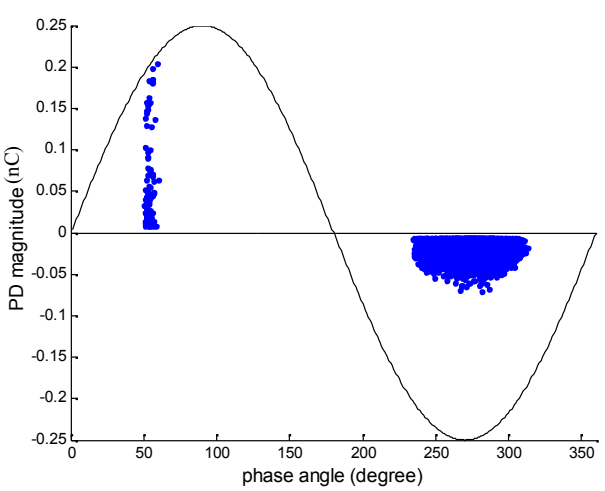

(b) $14 \mathrm{kV}$ applied voltage

Figure 7. PRPD patterns of corona discharge

\section{MEASUREMENT Results OF PSA PATterns (VOLTAGE DIFFERENCE)}

\section{A. Void discharge}

Figure 8 shows pulse sequential analysis (PSA) patterns using voltage difference between consecutive discharges of void discharge for different applied voltage amplitudes. In this figure, $\Delta U(n)$ equals to $U(n+1)$ minus $U(n)$ and $\Delta U(n-1)$ equals to $U(n)$ minus $U(n-1)$, where $U(n+1), U(n)$ and $U(n-1)$ are the applied voltage amplitude of the next, current and previous PD occurrences. From Figure 8, the maximum value of $\Delta U(n-1)$ and $\Delta \mathrm{U}(\mathrm{n})$ (unit in $\mathrm{kV}$ ) increase when the applied voltage is increased from $18 \mathrm{kV}$ to $24 \mathrm{kV}$ but the general pattern in the figures do not change significantly. The PSA patterns seem to be scattered but the concentration is higher along the positive $\Delta U(n)$ and negative $\Delta U(n-1)$ axes. A large $\Delta U(n)$ and $\Delta U(n-1)$, both negative and positive, indicates that consecutive PDs occur at the opposite polarity of the applied voltage. However, a small $\Delta U(n)$ and $\Delta U(n-1)$ indicates that consecutive PDs occur at the same polarity of the applied voltage. Therefore, these patterns indicate the sequence of PDs.

\section{B. Surface discharge}

Figure 9 shows PSA patterns using voltage difference between consecutive PDs of surface discharge for different applied voltage. When the applied voltage is increased from 3 $\mathrm{kV}$ to $6 \mathrm{kV}$, PDs occurring at the region of small $\Delta \mathrm{U}(\mathrm{n}-1)$ and $\Delta U(n)$ increase. These regions indicate that consecutive PDs are negative surface discharge, based on the PRPD patterns in
Figure 6. At one negative applied voltage cycle, many PDs can occur because high electric field region is wide on the material surface. Thus, the voltage difference between consecutive PDs is small. A larger $\Delta U(n)$ and $\Delta U(n-1)$ indicates that consecutive PDs are positive and negative surface PDs.

\section{Corona discharge}

Figure 10 shows PSA patterns using voltage difference between consecutive PDs of corona discharge for different applied voltage amplitude. There are more regions on the PSA pattern appear when the applied voltage is higher. In Figure $10 \mathrm{a}$, there is only one region of PD pattern, which is at small $\Delta \mathrm{U}(\mathrm{n})$ and $\Delta \mathrm{U}(\mathrm{n}-1)$. The level of the applied voltage amplitude for consecutive PDs is almost equal to each other, resulting in the difference between consecutive voltages is small. This is due to consecutive PDs occur at negative applied voltage.

However, the PSA pattern has four groups in Figure 10b. The top left corner group in the figure, which is large positive $\Delta \mathrm{U}(\mathrm{n}-1)$ and negative $\Delta \mathrm{U}(\mathrm{n})$ is due to the applied voltage polarity change between consecutive PDs. The centre group in the figure is small $\Delta U(n-1)$ and $\Delta U(n)$, indicating that the voltages between consecutive PDs are almost the same with each other. This group is due to consecutive PDs is negative corona discharge. The group on the most right side is small $\Delta \mathrm{U}(\mathrm{n})$ but large $\Delta \mathrm{U}(\mathrm{n}-1)$ while the bottom group is small

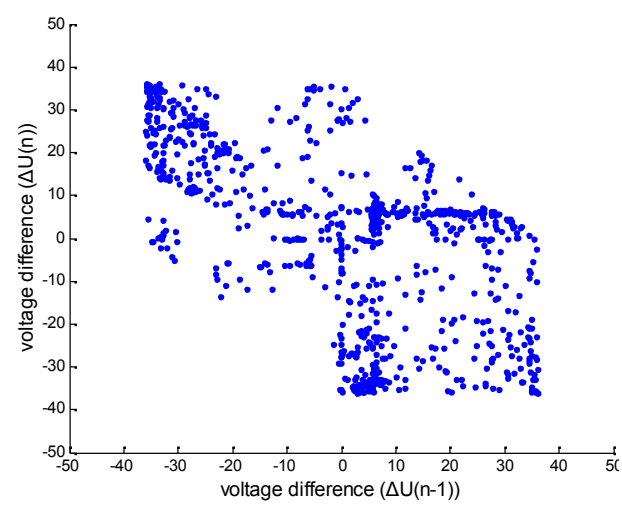

(a) $18 \mathrm{kV}$ applied voltage

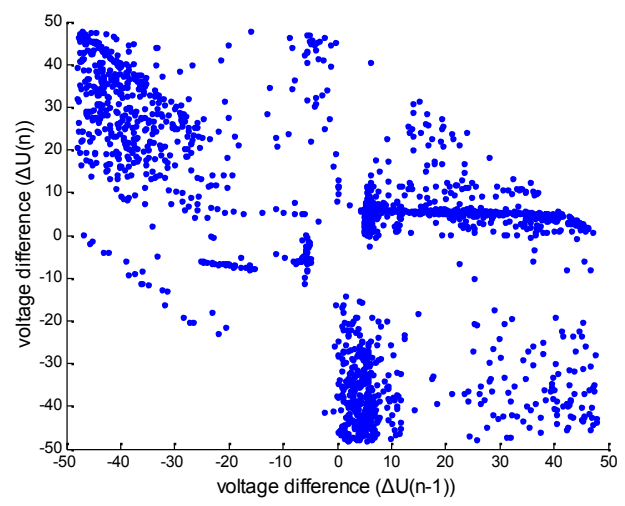

(b) $24 \mathrm{kV}$ applied voltage

Figure 8. PSA voltage difference patterns of void discharge 
$\Delta \mathrm{U}(\mathrm{n}-1)$ but large $\Delta \mathrm{U}(\mathrm{n})$. These two groups indicate that consecutive discharges occur at different polarity of the applied voltage, resulting in small and large voltage differences between consecutive discharges. In general, the PSA pattern of corona discharge is more concentrated at certain region rather than dispersed, unlike surface and void discharges.

\section{MEASUREMENT Results of PSA PATterns (Time DIFFERENCE)}

\section{A. Void discharge}

The PSA patterns using time difference between consecutive discharges of void discharge are shown in Figure

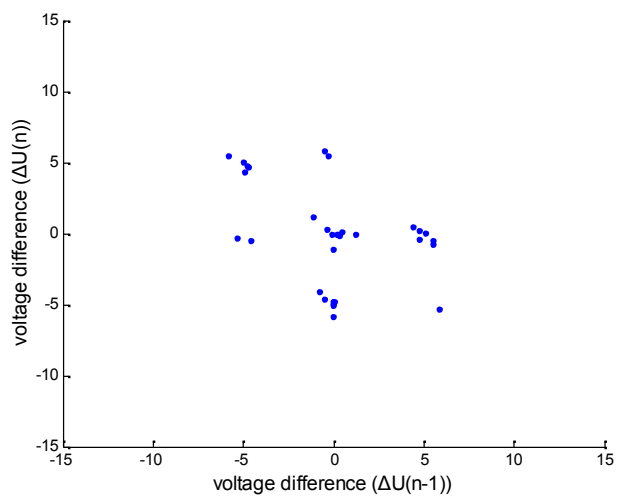

(a) $3 \mathrm{kV}$ applied voltage

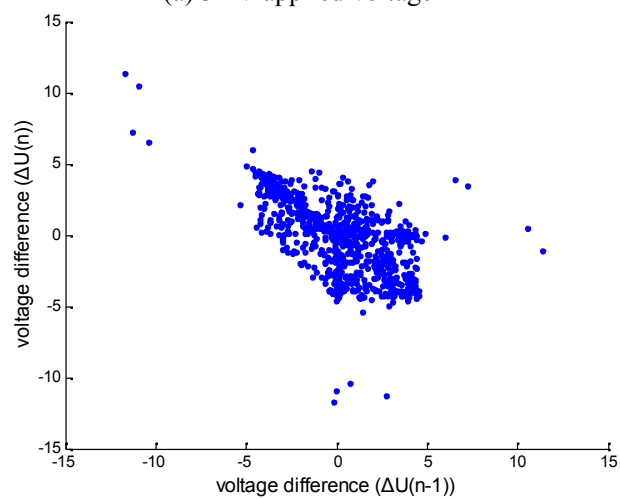

(b) $6 \mathrm{kV}$ applied voltage

Figure 9. PSA voltage difference patterns of surface discharge

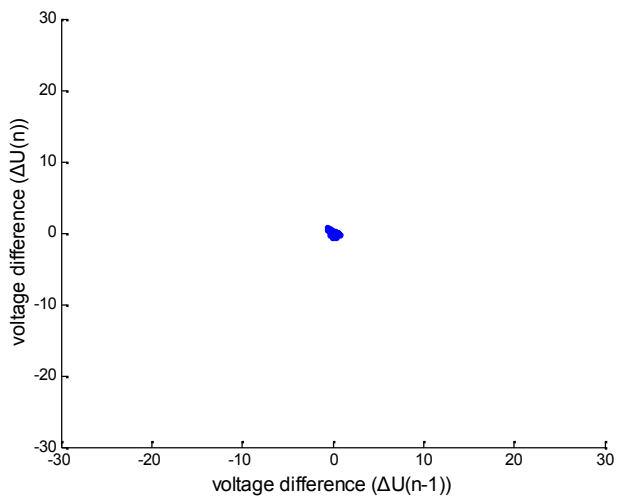

(a) $10 \mathrm{kV}$ applied voltage

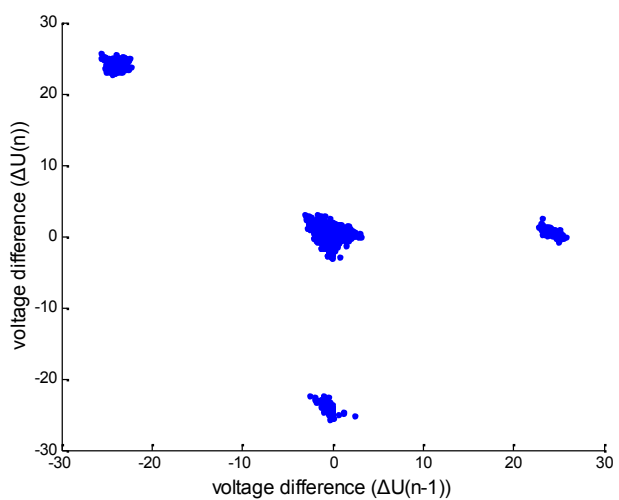

(b) $14 \mathrm{kV}$ applied voltage

Figure 10. PSA voltage difference patterns of corona discharge

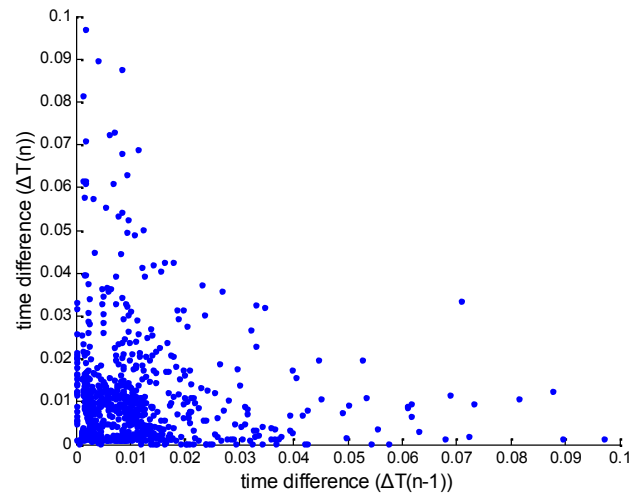

(a) $18 \mathrm{kV}$ applied voltage

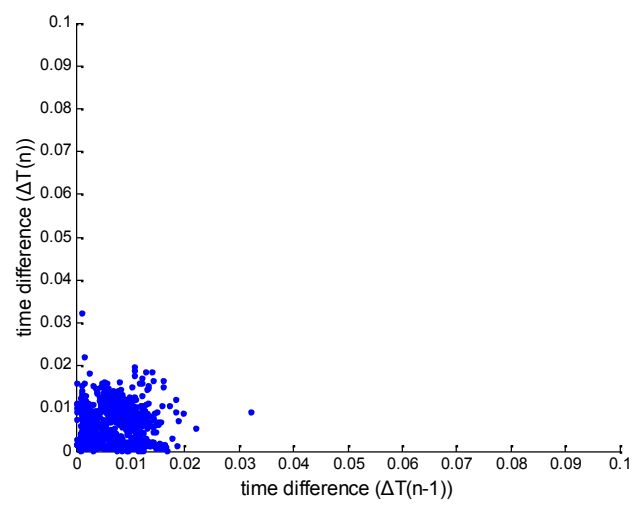

(b) $24 \mathrm{kV}$ applied voltage

Figure 11. PSA time difference patterns of void discharge

11 (unit in ms). At higher applied voltage, the time difference between consecutive discharges is shorter, where the pattern is concentrated near to zero value. This indicates that the number of PDs per cycle increases.

\section{B. Surface and corona discharges}

The PSA patterns between consecutive PDs of surface and corona discharges for different applied voltage amplitude are shown in Figures 12 and 13. The time difference between consecutive PDs decreases at higher applied voltage, indicating more PD events occur per applied voltage cycle. 
From the results of PRPD and PSA patterns that have been obtained, the main advantage and disadvantage can be identified from the two techniques, as shown in Table 1.

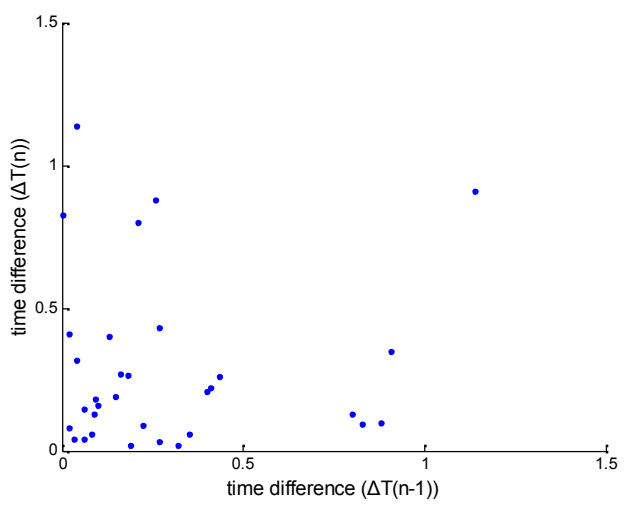

(a) $3 \mathrm{kV}$ applied voltage

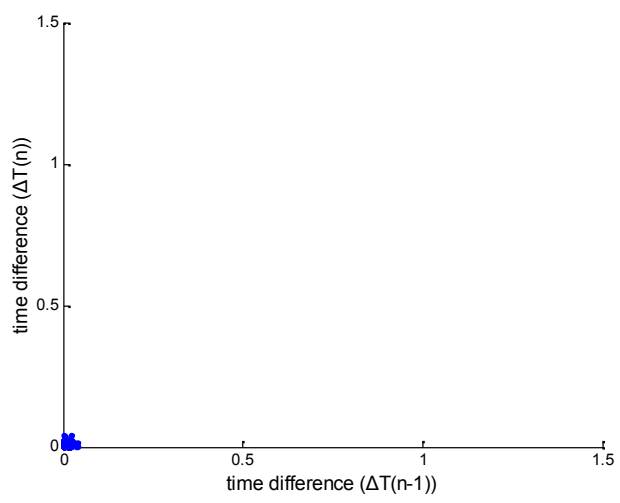

(b) $6 \mathrm{kV}$ applied voltage

Figure 12. PSA time difference patterns of surface discharge

TABLE I. COMPARISON BETWEEN PRPD AND PSA METHOD

\begin{tabular}{|l|l|l|}
\hline \multicolumn{1}{|c|}{ Method } & \multicolumn{1}{|c|}{ PRPD method } & \multicolumn{1}{c|}{ PSA method } \\
\hline Data recorded & $\begin{array}{l}\text { PD phase and charge } \\
\text { magnitude }\end{array}$ & $\begin{array}{l}\text { Time and voltage of PD } \\
\text { occurrence }\end{array}$ \\
\hline Advantage & $\begin{array}{l}\text { Statistical data can be } \\
\text { obtained from the pattern }\end{array}$ & $\begin{array}{l}\text { Sequence of PDs can be } \\
\text { identified }\end{array}$ \\
\hline Disadvantage & Sequence of PDs is lost & $\begin{array}{l}\text { Statistical data cannot be } \\
\text { obtained from the pattern }\end{array}$ \\
\hline
\end{tabular}

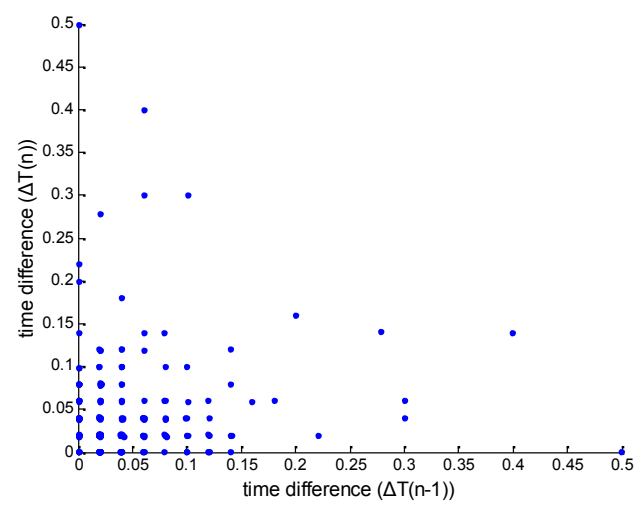

(a) $10 \mathrm{kV}$ applied voltage

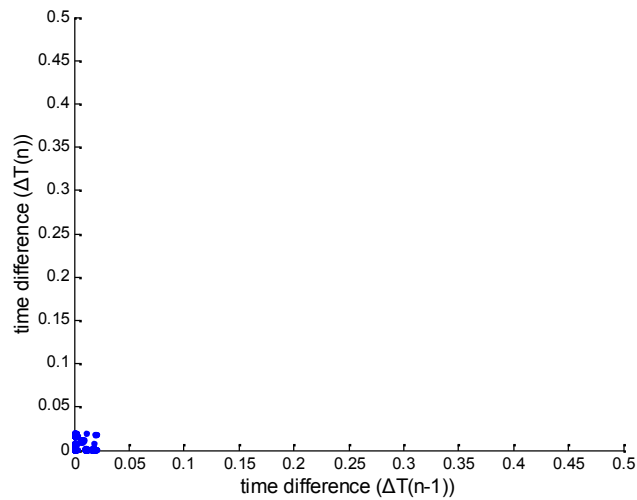

(b) $14 \mathrm{kV}$ applied voltage

Figure 13. PSA time difference patterns of corona discharge

\section{CONCLUSIONS}

Three types of PD have been analysed in this work using phase resolved partial discharge (PRPD) and pulse sequential analysis (PSA) techniques. Both methods were able to distinguish between three main PD types; they are void, surface and corona discharges. From PRPD patterns, statistical data of PD can be obtained but the sequence of PDs is lost. However, from PSA pattern, the sequence of PDs can be observed but the statistical data cannot be evaluated. Therefore, both methods have their own advantage and disadvantage. They are very useful in condition monitoring of high voltage insulation system in identifying different types of PDs.

\section{ACKNOWLEDGMENT}

The author thanks the University of Southampton, UK for providing the test facilities and the University of Malaya for supporting this work through the HIR research grant (Grant no: H-16001-00-D000048).

\section{REFERENCES}

[1] H. Illias, G. Chen, and P. L. Lewin, "Partial Discharge Behavior within a Spherical Cavity in a Solid Dielectric Material as a Function of Frequency and Amplitude of the Applied Voltage," IEEE Transactions on Dielectrics and Electrical Insulation, vol. 18, pp. 432-443, 2011.

[2] C. Forssen, "Modelling of cavity partial discharges at variable applied frequency," PhD Thesis, 2008.

[3] P. Rainer and B. Farhad, "Pulse Sequence Analysis - a diagnostic tool based on the physics behind partial discharges," Journal of Physics D: Applied Physics, vol. 35, pp. 25-32, 2002.

[4] H. Illias, G. Chen, and P. L. Lewin, "The influence of spherical cavity surface charge distribution on the sequence of partial discharge events," Journal of Physics D: Applied Physics, vol. 44, pp. 1-15, 2011.

[5] H. Illias, G. Chen, and P. L. Lewin, "Modeling of partial discharge activity in spherical cavities within a dielectric material," IEEE Electrical Insulation Magazine, vol. 27, pp. 38-45, 2011.

[6] H. Illias, G. Chen, and P. L. Lewin, "Partial Discharge within a Spherical Cavity in a Dielectric Material as a Function of Cavity Size and Material Temperature," IET Science, Measurement \& Technology, vol. 6 , pp. 52-62, 2012.

[7] E. Kuffel, W. S. Zaengl, and J. Kuffel, High Voltage Engineering: Fundamentals, 2nd ed.: Newnes, Butterworth-Heinemann, 2000. 